\title{
A alegoria da caverna e o atual panorama da política pública de saúde no modelo da atenção primária a saúde
}

The allegory of the cave and the current panorama of public health policy in the primary health care model

La alegoría de la cave y el panorama actual de la política publica de salud en el modelo de cuidado de salud primaria

Cinthya Luna Veloso de Lima ${ }^{1 *}$, Antonio Carlos de Souza Neto².

\begin{abstract}
RESUMO
Objetivos: Descrever e analisar as atividades dos profissionais de saúde dentro das Unidades Básicas de Saúde (UBS) e as atuais políticas públicas de saúde, observando evoluções e entraves para o desenvolvimento e fortalecimento do Sistema Único de Saúde e seus serviços de saúde, utilizando como parâmetro comparativo o "Mito da Caverna" de Platão. Revisão bibliográfica: O SUS tem passado por um grande período de desafios e vem tentado recuperar a confiança nas instituições e nas políticas públicas para seu fortalecimento e ampliação da produção do conhecimento em estratégias de gestão democrática. Os profissionais de saúde necessitam de informações sempre atualizadas e capacitações que promovam a dinâmica dialética, gerando discussões sobre o incremento nas políticas públicas de saúde, o que irá garantir sua consolidação. Conclusão: Através do conhecimento analítico e do compromisso dos profissionais, da gestão em saúde e da sociedade, poderemos ter um Sistema Único de Saúde mais organizado, participativo, acessível e resolutivo.
\end{abstract}

Palavras-chave: Políticas públicas de saúde, Gestão em saúde, Estratégia de saúde da família, Mito da caverna.

\begin{abstract}
Objectives: To describe and analyze the activities of health professionals within the Basic Healthcare Units (UBS) and current public health policies, observing developments and obstacles to the development and strengthening of the Brazilian Healthcare System and its healthcare services, using Plato's "Myth of the Cave" as a comparative parameter. Literature review: Brazilian Healthcare System SUS has been going through a great period of challenges and has been trying to regain confidence in its institutions and public policies for its strengthening and expansion of knowledge production in democratic management strategies. Health professionals need always up-to-date information and training that promotes dialectical dynamics, generating discussions about the increase in public health policies, which will ensure their consolidation. Conclusion: Through analytical knowledge and commitment of professionals, health management and society, we can have a more organized, participatory, accessible, and resolving Brazilian Healthcare System.
\end{abstract}

Keywords: Public health policies, Health management, Family health strategy, Cave myth.

${ }^{1}$ Universidade Estadual de Ciências da Saúde (UNCISAL), Maceió - AL.

*E-mail: cinthya_lunaveloso@yahoo.com.br

2Universidade de São Paulo (USP), São Paulo - SP.

SUBMETIDO EM: 3/2020 | ACEITO EM: 4/2020 | PUBLICADO EM: 6/2020 


\section{RESUMEN}

Objetivos: Describir y analizar las actividades de los profesionales de la salud dentro de las Unidades Básicas de Salud (UBS) y las políticas actuales de salud pública, observando la evolución y los obstáculos al desarrollo y fortalecimiento del Sistema Unificado de Salud y sus servicios de salud, utilizando el "Mito de la Cueva" de Platón, como parámetro comparativo. Revisión de la literatura: EI SUS ha estado pasando por un gran período de desafíos y ha estado tratando de recuperar la confianza en las instituciones y políticas públicas para su fortalecimiento y expansión de la producción de conocimiento en las estrategias de gestión democrática. Los profesionales de la salud necesitan siempre información y formación actualizada que promueva la dinámica dialéctica, generando discusiones sobre el aumento de las políticas de salud pública, lo que garantizará su consolidación. Conclusión: A través del conocimiento analítico y el compromiso de los profesionales, la gestión de la salud y la sociedad, podemos tener un Sistema Unificado de Salud más organizado, participativo, accesible y resolutivo.

Palabras clave: Políticas de salud pública, Gestión de la salud, Estrategia de salud familiar, Mito de la cueva.

\section{INTRODUÇÃO}

O Brasil vem passando por diversas transformações nas políticas públicas de saúde ao longo do tempo e, concomitantemente, os profissionais perdem o senso crítico de todos os processos que o rodeiam, para avaliar, planejar e executar suas atribuições de forma lógica, crítica, eficaz e capaz de trazer resultados que fortaleçam a política de estratégias desenvolvidas dentro do Sistema Único de Saúde (SUS) (MATIAS M e JUNIOR C, 2015).

No SUS, dois programas atuaram amplamente na década de 1990, seguindo o modelo sanitarista e médico-assistencialista: o Programa de Agentes Comunitários (PACS) e o Programa de Saúde da Família (PSF), que em 1994, passa a ser denominado de Estratégia de Saúde da Família (ESF) (GIOVANELLA L, et al., 2008). As primeiras equipes eram compostas por médicos, enfermeiros, auxiliares e agentes comunitários, englobando cirurgiões-dentistas e outros profissionais da saúde a partir do ano de 2000 (PAIM JS, 2008).

Os profissionais de saúde integram o SUS preparados apenas para realizar atividades laborativas em suas atribuições técnicas, desconhecendo a complexidade, diretrizes e organização, as normas de funcionamento e o financiamento do sistema, gerando conflito entre os preceitos e a realidade de trabalho. Torna-se interessante analisar o atual panorama do modelo de atenção à saúde praticado no Brasil sob um viés filosófico, valendo-se, por exemplo, de uma comparação com a alegoria da caverna (FARIA HX e ARAÚJO MD, 2010).

Ao imaginar o profissional de saúde nas Unidades Básicas de Saúde (UBS), é possível fazer alusão ao prisioneiro da caverna, segundo conta o mito conhecido como "Alegoria da Caverna", do livro "República", do filósofo Platão. Platão relata um prisioneiro que vê a possibilidade de sair da caverna, mas, acorrentado, só consegue ver o fundo dessa caverna devido à luz que emana de uma fogueira. Essa luz permite ao prisioneiro enxergar apenas projeções de sombras de estátuas, de pessoas e objetos, apresentando cenas e situações cotidianas. Ao ver essas sombras, o prisioneiro começa a nominá-las, analisando e julgando situações. Ao retornar à caverna, o prisioneiro transmite o conhecimento adquirido, mas é ridicularizado por seus companheiros, pois eles acreditam apenas na realidade vivida na caverna (SILVA CE, 2017).

Ao comparar o prisioneiro da obra de Platão com os profissionais que trabalham na saúde, notamos que as dificuldades enfrentadas pelo prisioneiro ao sair da caverna fazem alusão às dificuldades enfrentada pelos profissionais da saúde ao sair das UBS em busca de educação continuada. Além da falta de oferta de educação permanente, não conseguem informações fidedignas e espaços para o diálogo com outros profissionais do SUS, com gestores das esferas federal, estadual e municipal e com a sociedade. Ainda que o crescimento intelectual se torne dolorido aos profissionais, garante um olhar crítico e analítico do processo de trabalho e da organização nas práticas em saúde (FEUERWERKER L, 2005). 
Esse ambiente de escuridão intelectual reduz a percepção desses profissionais a respeito das políticas públicas de saúde. Em analogia à obra de Platão (1999), esses profissionais de saúde permanecem voltados ao trabalho individual, mantendo a hierarquização verticalizada e médico-centrada. Habituados à "caverna" (UBS) e às "correntes", representando as regras, acabam aceitando as imposições da gestão de saúde, ampliando disputas e conflitos entre profissionais e gestão e inviabilizando um melhor desenvolvimento no cumprimento das atividades na saúde (ALBUQUERQUE MIN, 2011).

A dificuldade de sair das UBS por vezes é negligenciada, mas quando o trabalho é esclarecido e prescrito a esses profissionais, retira-se as correntes do desconhecimento, despertando o conhecimento crítico e político, que viabiliza capacitações, treinamentos e pesquisas científicas, expondo a necessidade de discussões sobre a organização no processo de trabalho quando o profissional não está preparado e não tem o esclarecimento sobre o objetivo e os instrumentos para execução desse trabalho (BRITO E FREITAS JAS, 2006).

O trabalho realizado pelos profissionais na rede de Atenção Básica de Saúde (ABS) tem a finalidade de assistir a seus usuários, garantindo diagnósticos precoces de determinadas doenças, como câncer e doenças crônico-degenerativas, além de garantir qualidade, exatidão, reabilitação e recuperação. Porém, ainda é grande o número de profissionais que desconhecem o SUS, não percebendo o papel de resolutividade e cuidado humanizado, reafirmando as alegações de gestores a respeito da falta de qualidade nos atendimentos relacionada à diminuição dos repasses e de incentivos para aplicação na saúde. (GIOVANELLA L, et al., 2008).

A tendência de investimentos na compra de serviços no setor privado traz como uma consequência a precarização dos serviços de saúde oferecidos pelo setor público, tendo em vista que tais recursos poderiam ser investidos na ampliação da rede pública de saúde. Ofertar capacitações aos profissionais e gestores é trazer uma série de cobranças, compromissos e seriedade na aplicação do capital público. Assim, maior investimento, participação dos profissionais e da comunidade e comprometimento dos gestores de saúde resultará no fortalecimento do SUS, afastando assim o risco da privatização de setores da saúde, o que é considerado um retrocesso perante todos os avanços ocorridos durante décadas (SILVA CMTR, et al., 2017).

Vários modelos e políticas públicas de saúde foram implantados, destacando-se o modelo de assistência da ABS. A presente revisão sistemática tem como objetivos identificar a vivência dos profissionais do SUS, bem como as consequências da atuação nas UBS, através de uma análise das atuais políticas públicas de saúde a nível da Atenção Básica à luz do mito da "Alegoria da Caverna", descrevendo suas evoluções e entraves para o seu desenvolvimento e fortalecimento. Pretende-se comparar esse mito às atividades dos profissionais de saúde dentro das Unidades Básicas de Saúde, desvendando a necessidade desses profissionais participarem de capacitações, estabelecendo um compromisso em políticas públicas que garanta a continuidade da ESF.

\section{REVISÃO BIBLIOGRÁFICA}

\section{Políticas Públicas de Saúde}

Em 1990, o país assume uma agenda neoliberal: de um lado, o poder do Estado busca transformações na reestruturação produtiva e redução de custos com a elevação da produtividade e da competitividade, com a flexibilização do trabalho e a apropriação do fundo público vinculado à seguridade social objetivando a valorização e acumulação do capital vinculado a dívida pública (SALVADOR E, 2010). Do outro, há a luta para a ampliação do poder do SUS com a redução do poder do Estado (ALBUQUERQUE MIN, 2011).

O SUS conforma um modelo público de ações e serviços de saúde no Brasil, sendo implantado no início da década de 1990, após a promulgação de Lei Orgânica da Saúde (8080/90), e complementado pela lei no 8142, de 28 de dezembro de 1990. A partir desses marcos, o sistema de saúde toma uma nova forma nos entes governamentais, na prestação de serviços e na gestão do sistema de saúde, estabelecendo novos critérios com a participação dos gestores, prestadores de serviços privados, profissionais de saúde e usuários (BRASIL, 1988). 
Segundo Campos GWS (1992), inegavelmente, a década de 80 apresentou mudanças no modelo assistencial à saúde no país, particularmente por meio da Atenção Básica, desenvolvida em postos, ambulatórios e centros de saúde. A organização do processo de trabalho era baseada no trabalho em equipes vinculadas às famílias ou pacientes ou local de conveniência social (escolas, fábrica etc.). Pensava-se, com isso, em alternativas práticas que superassem o papel da clínica, que funcionava como um instrumento de reprodução da força de trabalho de forma distorcida, somente dispensando medicamentos, ou cometia iatrogenia ao tratar de problemas de ordem afetiva e social. A equipe trabalhava com a utopia de buscar condições facilitadoras à reprodução da vida de pessoas, com ponderações sobre o conflito entre expectativas socialmente definidas pelos usuários e às originadas dos saberes e práticas dos profissionais de saúde.

Com essa ideia, a Política Nacional de Atenção Básica (PNAB) define a Atenção Básica enquanto ações de promoção, proteção, prevenção de agravos e diagnóstico, visando também o tratamento, reabilitação e manutenção da saúde, tanto no âmbito individual como coletivo, garantindo de modo democrático a participação igualitária da população. $O$ trabalho em equipe passa a ser desenvolvido em território de responsabilidade da equipe para uma população adscrita, com responsabilidade sanitária desse território.

Essa prática tem como prioridade os usuários e sua dinâmica no território. Além disso, a Atenção Básica utiliza=se de tecnologias de alta complexidade e baixa densidade na resolutividade da assistência à saúde, devendo caminhar sempre orientada por princípios tais com: universalidade, equidade e igualitariamente, buscando aumentar a acessibilidade, o cuidado permanente e a participação social, e sendo o contato preferencial dos usuários com o sistema de saúde (BRASIL, 2017).

Com as reformas dos sistemas de saúde, na década de 1970 e seu ideário tão influente, houve um acúmulo do conhecimento que promoveu a utilização de novas práticas e outros tipos de formação dos recursos humanos de modo centralizado nos locais em que as propostas do movimento sanitarista ecoavam, cujas forças estavam centradas em questões mais gerais, no espaço das políticas e dos direitos dos pacientes a saúde (CONILL EM, 2008).

Portanto, na década de 1990, o Ministério da Saúde fortalece as ações de caráter preventivo com investimentos em programas de ações básicas como parte da estratégia de reorganização do próprio modelo de atenção, visando a promoção da saúde. A formulação do PSF foi estimulada por esses antecedentes e se materializou com a Portaria MS n.692 (GIOVANELLA L, et al., 2008), sendo porta de entrada preferencial do SUS, pois possui um espaço privilegiado da gestão do cuidado das pessoas e cumpre um papel estratégico na rede de atenção, servindo como base para o seu ordenamento e para a efetivação da integralidade.

\section{Profissionais da Estratégia de Saúde da Família}

Elaborado em 1991 pelo Ministério da Saúde, é publicado o primeiro Manual dos Agentes Comunitários de Saúde, denominado "O Trabalho do Agente Comunitário", para o treinamento no Programa Nacional de Agentes Comunitários (PNACS). Sendo realizado, em 1994, uma releitura do trabalho dos agentes comunitários de saúde, incluindo a família enquanto centro da produção dos cuidados em saúde (BRASIL, 2011).

Com a Portaria № 692/94, o PSF é instituído, tendo como propósito colaborar na organização do SUS e na municipalização. Porém, PACS e PSF nasceram de uma crise estrutural no setor saúde no Brasil vivenciada no modelo assistencial de saúde vigente por muitas décadas, demonstrando o distanciamento entre direitos garantidos em lei e capacidade efetiva do Estado de ofertar as ações e serviços públicos de saúde o mais próximo possível dos territórios socioeconômicos dos indivíduos, famílias e comunidades atendidos (SOUZA MF e HAMANN EM, 2009).

A partir de 1994, ocorre uma migração dos trabalhadores nos municípios, além de conflitos e insatisfações. A partir de então, a ABS passa a incorporar institucionalidade gradativamente, o que inclui uma gama de novos atores (gestores estaduais e municipais, profissionais de saúde, instituições formadoras e usuários), além da mobilização de recursos, que disputam politicamente a organização do sistema de saúde (BORGES CF e BAPTISTA TWF, 2010). 
No âmbito da atenção à saúde, o Ministério da Saúde, através da Portaria de oㅡ‥488/11, revisa as normas e diretrizes para organização das Equipes de Saúde da Família e PACS, o que traz importantes estratégias para o aprimoramento e consolidação do SUS, reorientando a assistência ambulatorial e domiciliar. Em 2000, ocorre o incentivo para incorporação dos profissionais da saúde bucal, inicialmente composta por médicos, enfermeiros, técnicos de enfermagem e ACS (BRASIL, 2011).

$\mathrm{Na}$ atualidade, após a aprovação da Política Nacional de Atenção Básica (PNAB), regida pela portaria no 2.436/2017, é estabelecida uma revisão de diretrizes para a organização da Atenção Básica $(A B)$ no âmbito do SUS. A PNAB passa a ser resultado cumulativo de um conjunto de atores envolvidos historicamente com o desenvolvimento e a consolidação do SUS, como movimentos sociais, população, trabalhadores e gestores das três esferas do governo (BRASIL, 2017).

O SUS incorporou trabalhadores de saúde, usuários e gestores que precisam de políticas públicas de saúde mais consistentes. Entretanto, a sua edificação e fortalecimento depende decisivamente de profissionais que possuam habilidades para o cumprimento de atividades básicas da prática profissional, para o preenchimento de lacunas que ocasionam debilidades na assistência à saúde e na articulação da produção políticas de saúde (FIGUEIREDO PP, et al., 2010).

Atualmente, no contexto da política pública de saúde, o SUS enfrenta grandes desafios para recuperar a confiança nas instituições e na política para ampliação da produção do conhecimento a respeito das estratégias de gestão democrática, num processo de trabalho em saúde com diferentes possibilidades de construção da rede de atenção à saúde e na produção de novas tecnologias de cuidado orientadas às necessidades da saúde dos usuários, resgatando o espaço da cidadania (MEHRY EE, 2005).

Pode-se afirmar que as políticas públicas de saúde são um resultado cumulativo de pontos favoráveis e desfavoráveis que nos levam a bons resultados e muitas expectativas, após uma luta contínua de movimentos sociais, população, trabalhadores e gestores. O trabalho em saúde, por ser de grande complexidade e dinamicidade, é executado sem orientações necessárias gerando confronto entre o trabalho prescrito e o real. (FARIA HX e ARAÚJO MD, 2010).

Para as políticas de saúde, toma destaque a Atenção Básica através da ESF, que se configura na intervenção de maior visibilidade e impacto do país. A expansão vem ocorrendo com tendência crescente: em 2002, havia mil equipes implantadas em mais de quatro mil municípios, apresentando uma cobertura de mais de 50 milhões de pessoas e uma estimativa de aproximadamente 170 mil postos de trabalho diretamente na condução da estratégia, sem contar pessoal de apoio administrativo. Até fevereiro de 2011, haviam sido implantadas 31.883 equipes de saúde da família, em 5.290 municípios (de 5.564 municípios brasileiros), com cobertura de aproximadamente $62,8 \%$ da população brasileira (BRASIL, 2011).

Por outro lado, vem ocorrendo uma precarização das condições de trabalho, percebida através da falta de materiais e pela precária infraestrutura, que aumenta o improviso das ações realizadas, indicando práticas de saúde sem planejamento entre os sujeitos, trabalhadores e usuários do serviço. Ou seja, os trabalhadores reconhecem que estão em contextos socioambientais vulneráveis, o que constitui a razão do descontentamento profissional, além da infraestrutura inadequada e falta de reconhecimento institucional, relacionadas aos fatores de desgaste e adoecimento do trabalhador da saúde (FIGUEIREDO PP, et al., 2010).

\section{Analogia com o Mito da Caverna}

Ao fazer uma analogia com o ideário pedagógico, Platão, no livro VII da República, utiliza-se do mito da Alegoria da Caverna, que contém o cerne de seu pensamento educacional. Busca-se transpor sua analogia aos profissionais de saúde que atuam nas UBS, aos gestores municipais e outros atores do processo de edificação das políticas públicas de saúde.

Refletindo por volta de 427-347 a.C., Platão, diz que o homem deve ter uma educação suficiente e orientada para alcançar a realidade em sua profundidade e veracidade. Essa necessidade do homem o conduz às aspirações divinas e à verdadeira resignação. Ao ofertar a educação, distanciando os profissionais 
da ideia de incapacidade, estes despertam em potencial para o desenvolvimento das suas atribuições dentro das UBS, mantendo os índices e metas tão cobradas pela gestão em saúde e aumentando o respeito aos usuários (HELMAN C, 2003).

Na Alegoria da Caverna, Platão nos faz imaginar prisioneiros acorrentados em uma caverna e sem mobilidade para mexer pernas e pescoço, olhando apenas para frente. No exterior da caverna subterrânea, existe um caminho elevado, formando uma parede pequena onde são projetadas sombras graças à luz de uma fogueira. Os prisioneiros apenas ouvem o eco de suas próprias vozes e as vozes de pessoas que andam silenciosamente no exterior da caverna. As sombras tornam-se para eles a realidade, assim como o próprio interior da caverna (SILVA CE, et al., 2017).

Analogicamente, os profissionais que não buscam sair da "caverna" acabam por ser prisioneiros e não conseguem enxergar que as sombras projetadas pela gestão são distorcidas e que esses profissionais perdem paulatinamente sua capacidade criativa e intelectual e avaliação crítica, pela falta de formações acadêmicas mais direcionadas a PNAB, gerenciamento, financiamento e funcionamento. Essa centralização dos processos políticos e a fragmentação do trabalho levam os profissionais ao desinteresse, à alienação e à desresponsabilização em relação aos resultados finais (CAMPOS GWS,1992; MEHRY EE, 2002), além da falta de retroalimentação por parte da gestão dos indicadores produzido nas UBS (ALBUQUERQUE MIN, 2011).

Ao sair da caverna, o prisioneiro ficaria ofuscado pela luz. Mas quando é obrigado a redirecionar o pescoço, sentiria muitas dores ao realizar o movimento, não conseguindo reconhecer os formatos dos objetos e associá-los às sombras antes vistas no interior da caverna. Porém, com o passar do tempo, o prisioneiro ainda confuso conseguiria ver e identificar que as sombras antes vistas não condiziam com os objetos e afirmaria que as sombras são mais verdadeiras. Quando liberto, o prisioneiro abandona as amarras e a enfermidade da ignorância (SILVA CE, et al., 2017).

O mesmo processo pode ser evidenciado pelos profissionais que buscam manter um diálogo, analisando e debatendo técnicas e experiências vividas, aperfeiçoando-se e se qualificando, adquirindo capacidade de realizar críticas, gerando uma série de informações, edificando um conhecimento com base empírica e científica. Quando saem das UBS, trazem valores que deveriam ser agregados na melhoria do trabalho intra e intersetorial. Porém, devido aos grandes interesses capitalistas, do neoliberalismo, que influenciaram desde o começo a história da saúde, tanto os profissionais como a comunidade e demais atores são excluídos de todo processamento formador e facilitador.

Ao pensar no prisioneiro, retirado à força da caverna e obrigado bruscamente a subir em direção à saída da caverna, Platão nos leva a vislumbrar que a luz do conhecimento inteligível faz o homem sair da acomodação, deixando-o irritado a princípio por não conseguir discernir a verdade. Caso retornasse à caverna, deixaria de ver a luz, novamente se acostumaria com a escuridão e, ao dialogar com os outros prisioneiros, provocaria risos e ouviria ao retornar a caverna que ficou cego e que essa experiência não vale a pena. Por fim, acabaria morto se tentasse os libertar e os conduzir à luz (SILVA CE, et al., 2017).

A educação permanente para os profissionais da saúde estimula o diálogo, distanciando-os da apatia, desmotivação e submissão a outros profissionais. Ao enxergar sua capacidade de participantes ativos no processo de trabalho, extingue-se a rigidez hierárquica, viabilizando canais legítimos para sua participação efetiva, tornando as UBS um ambiente propício de aproximação, preservando o coletivo, afastando dos profissionais sentimentos de inferioridade (ALBUQUERQUE MIN, 2011).

Segundo Platão (1999), o mito da caverna amplia o olhar para a história da humanidade influenciada pela visão ocidental, o que constitui o presente e o que constituirá os futuros acontecimentos na história. Definindo a verdade como a exatidão da representação, o homem passa a ter suas ideias, na observância dos seus valores. Esses valores são estabelecidos e aceitos trazendo, de modo geral, a real interpretação do mundo de acordo com suas ideias (PLATÃO, 1999). Nesse pensamento filosófico, reflete-se a respeito de um ponto comum em que sejam compartilhados valores, conhecimentos, informações, deixando de existir uma 
preocupação dos gestores, profissionais e comunidade com a atuação e as metas a cumprir, exigidas e comprovadas através do preenchimento diário e sistemático (ALBUQUERQUE MIN, 2011).

Paviani J (2008), ao citar Platão, reafirma que a mudança de comportamento só poderá ser alcançada através da dialética. É através da dialética, que ao sair da caverna - UBS -, alcançamos o conhecimento inteligível, com perguntas e respostas inteligentes (arte do bem falar), sendo atualizada em cursos de especialização, educação permanente e continuada, dos profissionais da saúde, o que garante resoluções e participação ativa de todos em questionamentos a respeito do financiamento, compra de insumos e equipamentos, além da sua distribuição igualitária (PAVIANI J, 2008).

A educação para Platão (1999) é o meio prioritário para o homem conseguir vislumbrar a posição correta das coisas, já que traz consigo o modo fácil e eficaz para olhar a sua volta, não de onde virá algo mas olhar os meios para alcançar o que é visto (PLATÃO, 1999).

Sem dúvidas, Platão se faz visceralmente atual, propondo de maneira pedagógica e filosófica, interpelando-nos a questionar acerca da realidade, sem o frágil verniz das projeções. Seu convite à libertação total do ser humano ecoa ainda em nossas mentes, agregando com a educação libertária contemporânea, raiz de todos os movimentos que participam do fortalecimento do SUS e suas políticas públicas de saúde.

\section{CONSIDERAÇÕES FINAIS}

O caminho dialético rumo ao conhecimento inteligível e à luz da verdade torna nobre o homem liberto da acomodação e da ignorância e pode ser aplicado às limitações para consolidação e amadurecimento de políticas públicas de saúde, em que todos possam exercer o seu papel como profissional, na gestão em saúde e na comunidade e sociedade. A analogia à "Alegoria da Caverna" se faz atual, demonstrando que a educação permanente torna os profissionais capazes, resolutivos e dinâmicos no processo de trabalho, fortalecendo as ações de promoção, proteção e prevenção na Atenção Básica de Saúde e, assim, favorecendo a sociedade.

\section{REFERÊNCIAS}

1. ALUQUERQUE MIN. Estratégia de Saúde da Família: um estudo sobre o processo e as condições de trabalho dos trabalhadores de saúde. Tese (Doutorado em Serviço Social) - Centro de Ciências Sociais e Aplicadas, Universidade Federal de Pernambuco, Recife, 2011, $244 \mathrm{p}$.

2. BORGES CF, BAPTISTA TWF. A Política de Atenção Básica do Ministério da Saúde: refletindo sobre a definição de prioridades. Trabalho, Educação e Saúde. 2010;8(1): 27-53.

3. BRITO E FREITAS, JAS. Ensaios literários e a dimensão tácita do conhecimento nas organizações. In: Encontro Nacional De Estudos Organizacionais, 3, Porto Alegre: ANPAD, 2006.

4. BRASIL. Constituição. Constituição da República Federativa do Brasil. Brasília, DF: Senado Federal, 1988.

5. BRASIL. Ministério da Saúde. Secretaria de Atenção à Saúde. Departamento de Atenção Básica. Portaria o 2.436, 21 de setembro de 2017. Aprova a Política Nacional de Atenção Básica, estabelecendo a revisão de diretrizes para a organização da Atenção Básica, no âmbito do Sistema Único de Saúde (SUS). Brasília, DF: Ministério da Saúde, 2017.

6. BRASIL. Ministério da Saúde. Secretaria de Atenção à Saúde. Departamento de Atenção Básica. Portaria no 2.4888 , 21 de outubro de 2011. Aprova a Política Nacional de Atenção Básica, estabelecendo a revisão de diretrizes e normas para a organização de Atenção Básica, para a Estratégia Saúde da Família (ESF) e o Programa de Agentes Comunitários de Saúde (PACS). Brasília, DF: Ministério da Saúde, 2011.

7. CAMPOS GWS. A reforma da reforma: repensando a saúde. São Paulo: Hucitec; 1992.

8. CONILL EM. Ensaio histórico-conceitual sobre à Atenção Primária: desafios para a organização de serviços básicos e da Estratégia Saúde da Família em centros urbanos no Brasil. Cadernos de Saúde Pública. 2008;24(Supl. 1): S7S27.

9. FARIA HX, ARAÚJO MD. Uma perspectiva de análise sobre o processo de trabalho em saúde: produção do cuidado e produção de sujeitos. Saúde e Sociedade. 2010;19(2): 429-439.

10. FIGUEIREDO PP, et al. Processo de trabalho da Estratégia Saúde da Família: a concepção de gestão que permeia o agir da saúde. Physis Revista de Saúde Coletiva. 2010;20(1): 235-259.

11. FEUERWERKER L. Modelos tecnoassistenciais, gestão e organização do trabalho em saúde: nada é indiferente no processo de luta para a consolidação do SUS. Interface (Botucatu) [online]. 2005;9(18): 489-506.

12. GIOVANELLA L, et al. Políticas e Sistema de Saúde no Brasil. Rio de Janeiro: Ed. Fiocruz, 2008; 1100 p.

13. HELMAN C. Cultura, saúde e doença. Porto Alegre: Ed. Artmed; 2003.

14. MATIAS M, JUNIOR C. 2015. SUS ontem e hoje: Perdemos a luta por um sistema de saúde universal? In: CARTA Maior, o portal da esquerda. Brasil. 
15. MEHRY EE. Engravidando as palavras: caso da integralidade. In: PINHEIRO R, MATOS RA. (Org.) Construção social da demanda. Rio de Janeiro: IMS-UERJ/Abrasco; 2005.

16. MEHRY EE. Saúde: a cartografia do trabalho vivo em ato. São Paulo: Hucitec; 2002.

17. PAIM JS. Modelos de Atenção à Saúde no Brasil. In: GIOVANELLA L, et al. Políticas e Sistema de Saúde no Brasil. Rio de Janeiro: Ed. Fiocruz, 2012; 1100 p.

18. PAVIANI, J. Platão e a Educação. Belo Horizonte: Autêntica, 2008; 128 p.

19. PLATÃO. As Leis - incluindo Epinomis. Tradução de Edson Bini. Bauru: Edipro; 1999.

20. SALVADOR E. Fundo Público e Seguridade Social do Brasil. São Paulo: Cortez; 2010.

21. SILVA CE, et al. A educação do homem segundo o ideário pedagógico de Platão: uma abordagem a Alegoria da Caverna. Cadernos da Unicamp. 2017;16(25): 7-26.

22. SILVA CMTR, et al. A Privatização do Sistema Único de Saúde via a complementariedade do setor privado ao público. VII Jornada Internacional Políticas Públicas. São Luís, MA; 2017.

23. SOUSA MF, HAMANN EM. Programa de Saúde da Família no Brasil: uma agenda incompleta? Ciência e Saúde Coletiva. 2009;14(Supl.1): 1325-1335. 